\title{
Formation of metal oxides based surface nanolenses and their optical propertie
}

Laraib Syeda*1, Carmen Van Horn*1, Jiasheng Qian ${ }^{1}$, Xuehua Zhang ${ }^{1}$

${ }^{1}$ Department of Chemical and Material Engineering, University of Alberta

${ }^{*}$ Contributed equally

\section{Abstract}

An emerging resource in the production of renewable energy are nanolenses, due to their unique optical properties. Their ability to refract light makes it possible for them to focus light and convert it into other forms of energy; which reduces the need for burning fossil fuels.

The formation of nanolenses occurs due to the process of solvent exchange. In this process, different concentrations of a ternary mixture comprising of oleic acid, water, and ethanol are used to create an ideal formation of nanodroplets. A mixture of iron (IV) chloride and manganese chloride is then washed over the droplets to create the droplet's shell. The droplets are then annealed at $300^{\circ} \mathrm{C}$ in order to remove all excess liquid, leaving behind the hollow nanolenses. This process was carried out on two different substrates, silicon wafer and glass with similar results transpiring on both.

After various trials, it can be concluded that the ideal concentration ratio of oleic acid/water/ ethanol is 4.25/30/70, as the lenses are homogeneous in size, volume, and distribution. Allowing them to remain intact through the annealing process. These findings can be applied to further studies in the use of nanolenses concerning light refraction, and the consequential production of renewable energy.

Key words:

nanolens, nanodroplets, optical properties, fluid cell, surface nanolens

Cite as: : Syeda, L., Van Horn, C., Qian, J., Zhang, X. 2019. Formation of metal oxides based surface nanolenses and their optical properties. Alberta Academic Review, Vol 2 (2) 73-74, WISEST Special Issue (non peer-reviewed), DOI 10.29173/aar72. 
Syeda et al., 2019

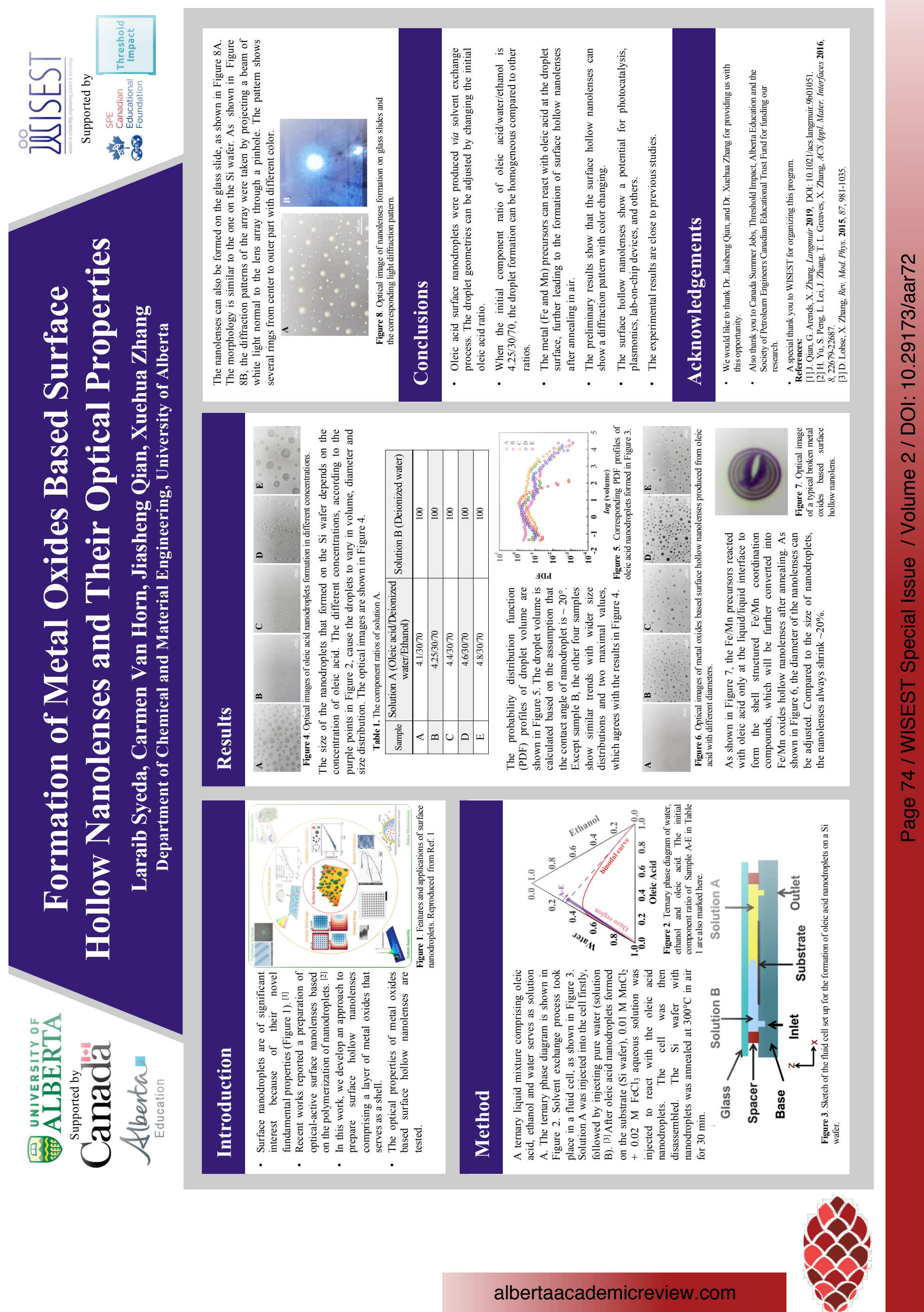

